\title{
Numerical Study of the High Speed Compressible Flow with Non-Equilibrium Condensation in a Supersonic Separator
}

\author{
Liu Xingwei, Liu Zhongliang, and Li Yanxia
}

\begin{abstract}
The present work is mainly focus on the internal flow in a supersonic separator. In order to explore the separation mechanism and heat transfer inside the separator, a fully three-dimensional numerical dynamic model with consideration of such effects as strong swirling turbulent and non-equilibrium condensation process is established. The numerical model is conducted to determine the dominant factors on the separation performance. The mixture of methane and water liquid are chosen as working fluid. The numerical model results are verified through comparison with experimental data obtained from the literature and the results demonstrated good agreement between the two ways. By using the proposed numerical model, the distribution of pressure, temperature, velocity, droplet nucleation rate and Mach number are investigated. Based on the established numerical model, the swirling intensity of the outlet of straight tube versus the swirling length ratio L5/L4 (L5 is the length of straight tube, L4 is the length of the swirling flow generator, in this paper is $25 \mathrm{~mm}$ ) are also studied. The optimal value of L5/L4 should be 8 with the pressure loss ratio specified 0.71 . Furthermore, the simulation results can provide a foundation for the analysis of flow characteristic caused by the supersonic speed and nucleation phenomenon and also a basis for the design and geometrical optimization of the supersonic separator.
\end{abstract}

Index Terms-Supersonic separator, gas-liquid two phase model, condensation process, flow field.

\section{INTRODUCTION}

Considering that conventional fossil fuel resources are being exhausted and the continuously increasing energy demand, exploring a sustainable alternate energy source is essential and urgent. Natural gas is one of the most important sources of energy in the world, which generally accepted as a potential strategic energy form for sustainable development. Currently, significant challenges need to be overcome in the commercial exploitation and processing of natural gas especially in China [1]. Thus, it is necessary to develop advanced technology to improve utilization efficiency of natural gas. The primary innovation step should focus on the dehydration technology, which urges researchers to pay more attention on exploring advanced separation technology.

The supersonic separation is a promising new technology to remove water liquid and gaseous components out of a mixed natural gas [2]-[5]. Compared to the traditional

Manuscript received July 1, 2014; revised August 28, 2014.

The authors are with Beijing University of Technology, College of Environmental and Energy Engineering,100124, Beijing, China (e-mail: Liuzhl@bjut.edu.cn). dehydration technology, the main virtue of the supersonic separation technology is the small size and flexible structure. Therefore, supersonic separator can be used to remove condensable vapors such as water or natural gas liquids (NGL) from a gas stream in order to lower the (water or hydrocarbon) dew point of the gas or strip the gas with heavy hydrocarbons which can generate additional investment. This new technology is suitable for unmanned operations, especially for the oil and gas field in the offshore, desert and remote areas [6]. The first team known to carry out investments on supersonic separators was an engineering group from the Netherlands that is now affiliated with Twister BV [7]. At the same time, a group of Russian specialists also work on the supersonic separator, and named them $3 \mathrm{~S}$ technology [8]. Experimental testing system was constructed by Liu [9] to fully analyze the process of dew point depression in supersonic flows. A range of field test investigations were conducted to predict the actual effect of supersonic dehydrating technology in SINOPEC Shengli Oilfield [10]. It has proved that this new supersonic dehydration technology for natural gas can not only reduce the dew point of natural gas but also recover light hydrocarbon at the same time. However, the present test results still indicate that the separation efficiency of the supersonic separator is far from satisfactory effect.

For the further research on the supersonic separator, the ability to explore the internal flow mechanism is significant. The flow in the supersonic separator is multi-component, strong swirling and also with non-equilibrium phase condensation. In current experimental conditions, obtain the whole realizable flow field is difficult or even impossible. Fortunately, numerical simulations can reduce research costs and provide the convenience in side of discovering new flow phenomena, recognizing flow details, extending research topics, and so forth, and with the rapid development of computers, numerical methods become more and more promising in understanding gas-liquid two phase flow with high speed phenomenon inside the supersonic separator. In the supersonic separation field, many numerical works have been done by researchers. For example, Malyshkina et al. [11], [12] presented their calculation results with two-dimensional Euler model of the natural gas supersonic swirling flow inside a supersonic separator. Jiang et al. [13] developed a mathematical model to investigate the one-dimensional transonic flow of two-component gas mixture with spontaneous condensation. But his researches only focus on Laval section of the supersonic separator. Bao et al. [14] established a mathematical model for phase equilibrium prediction of 
multi-component gas separation process inside a supersonic separator. Jassim et al. [15], [16] adopted computational fluid dynamics technique to study behavior of high-pressure natural gas in supersonic nozzles. Rajaee Shooshtari, S. H [17] proposed a novel mass transfer approach to develop a reliable model for estimation of liquid droplet growth rate for binary and multicomponent systems. Wen et al. [18] studied the separation efficiency using Discrete Particle Method (DPM). It is convenient to execute the calculation of the gas-droplets two-phase flow with discrete phase by using the model of particle group trajectory. Ma et al. [19] studied the performance of inner-core supersonic gas separation device with droplet enlargement method. In his model, the gas phase was treated as continuous medium and the particles as the discrete medium.

As mentioned above, Most of the literature only concerned with the basic structure and preliminary test aspects of this device and not take into account the gas dynamics mechanism and condensation aspects. Thus, establishing an accurate numerical model for high speed process of two-phase flow with non-equilibrium condensation within the supersonic separator will provide a great guidance for structure design, furthermore, can provide a reliable method to predict the dehydration efficiency of the supersonic separator.

Thus, in order to demonstrate the acceptable flow pattern and find the creditable explanation of separation mechanism in supersonic separator, this article established a 3D computational model based on the compressibility and strong swirling characteristics coupled with the gas-liquid two phase model and non-equilibrium condensation model which take into account the nucleation process and droplet growth process. Using this numerical model, we explore different parameters distribution within the supersonic separator.

\section{DESCRIPTION OF THE SUPERSONIC SEPARATOR}

The supersonic separator is mainly composed of three sections: a Laval nozzle (include convergence part and divergence part), a straight tube integrated with a cyclone (include swirling flow generator), and diffuser part, as shown in Fig. 1. In the Laval nozzle part, the mixture gas adiabatically expands, velocity increases and temperature drops, to promote the condensable gas with formation of droplets. After that, the mixture of gas and liquid or even solid phase is introduced into the straight tube. In the straight tube, centrifugal effects generated by the swirling flow generator composed of twist vanes drive the droplets towards the wall of the working section. Condensed liquid droplets are separated from main gas flow in the working section. The remaining dry gas stream flows into the diffuser in which about $50 \%-80 \%$ of the initial pressure is recovered. The wet gas can be released or used for other purposes.

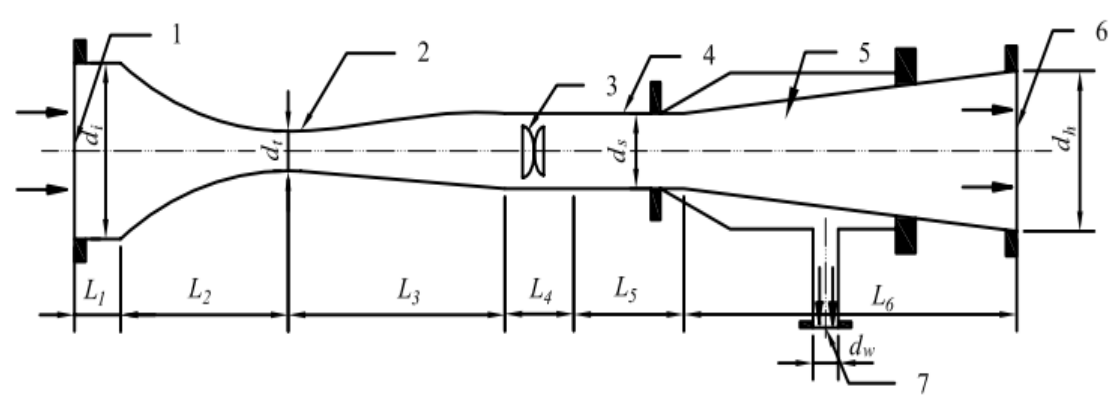

1-Saturated feed gas inlet; 2-Laval Nozzle; 3-swirling flow generator; 4-straight tube; 5-diffuser section; 6-dry gas outlet; 7-wet gas and slip-gas outlet Fig. 1. Geometry structure of the supersonic separator.

The velocity distribution from the orifices and the temperature distribution of the air jet were estimated by performing fluid analysis using a three-dimensional (3D) finite element method (FEM) with FLUENT 14.0 software. The analyses were estimated based on varying pressure.

\section{A. Numerical Methods}

The flow in the supersonic separator is assumed compressible and strong turbulent, the condensation process is also considered, relative governing equations of the two phases are given as follows:

\section{B. Gas Phase Control Equations}

Continuity equation

$$
\frac{\partial \rho_{g}}{\partial t}+\nabla \cdot\left(\rho_{g} \vec{v}_{g}\right)=\dot{m}
$$

Momentum equation

$$
\begin{array}{r}
\frac{\partial\left(\rho_{g} \vec{v}_{g}\right)}{\partial t}+\nabla \cdot\left(\rho_{g} \vec{v}_{g} \vec{v}_{g}\right)=-\nabla p+\rho_{g} \vec{g} \\
+\nabla \cdot \overline{\bar{\tau}}_{g}+\frac{\rho_{g} y}{\tau_{r l}}\left(\vec{v}_{l}-\vec{v}_{g}\right)-\vec{v}_{g} \dot{m}
\end{array}
$$

Energy equation

$$
\begin{array}{r}
\frac{\partial\left(\rho_{g} E\right)}{\partial t}+\nabla \cdot\left[\vec{v}_{g}(\rho E+p)\right]=-\dot{m}\left(h_{t}-h_{l g}\right) \\
+\nabla \cdot\left(k_{\text {eff }} \nabla T+\overline{\bar{\tau}}_{\text {eff }} \vec{v}_{g}\right)
\end{array}
$$

Gas state equation

The Virial equation is applied, and its universal form is given as follows:

$$
p=\rho_{g} R T_{g}\left(1+B \rho_{g}+C \rho_{g}^{2}\right)
$$


where $B$ and $C$ are second and third Virial coefficient, respectively.

\section{Liquid Gas Control Equation}

Continuity equation

$$
\frac{\partial\left(\rho_{l} y\right)}{\partial t}+\nabla\left(\rho_{l} y \vec{v}_{l}\right)=\dot{m}
$$

Momentum equation

$$
\begin{aligned}
\frac{\partial\left(\rho_{l} y \vec{v}_{l}\right)}{\partial t} & +\nabla \cdot\left(\rho_{l} y \vec{v}_{l}\right)=\rho_{l} y g_{i}+\nabla \cdot \overline{\bar{\tau}}_{l} \\
& +\frac{\rho_{l} y}{\tau_{g l}}\left(\vec{v}_{g}-\vec{v}_{l}\right)-\left(\vec{v}_{g}-\vec{v}_{l}\right) \dot{m}
\end{aligned}
$$

Droplet species equation

$$
\frac{\partial\left(\rho_{l} y\right)}{\partial t}+\nabla \cdot\left(\rho_{l} y \vec{v}_{l}\right)=\rho_{l} J
$$

Due to the existence of the water vapor, in the supersonic separator two significant processes will take place, one is non-equilibrium condensation nucleation and the other one is the droplet growth. It is extremely important to predict the two processes of nucleation and droplet growth accurately for the numerical simulation on the supersonic two phase flow. In this study, the description of the mass generation rate $\dot{m}$ provided by Yang [20] is chosen for the nucleation model, which indicate the sum of mass increase due to condensation and due to growth/demise of the formed droplet. The equations to express the condensation phenomenon can be written as follows.

Droplet mass equation

$$
\dot{m}=(1-y) J \rho_{l} \frac{4 \pi r_{c}^{3}}{3}+4 \pi r^{2} \frac{\partial r}{\partial t} \rho_{l} N
$$

where $N$ is the number density for droplets, and the $r_{c}$ denotes the critical droplet radius [13], which is based on the classical nucleation theory and can be written as:

$$
\begin{aligned}
& r_{c}=\frac{2 \sigma}{\rho_{l} R_{g} T_{g}\left[f_{1}+f_{2}+f_{3}\right]-p_{s}(S-1)} \\
& f_{1}=\ln \frac{\rho_{g}}{\rho_{s}}, f_{2}=2 B\left(\rho_{g}-\rho_{s}\right), f_{3}=\frac{3 C}{2}\left(\rho_{g}^{2}-\rho_{s}^{2}\right)
\end{aligned}
$$

The droplet nucleation rate $J$ and droplet growth rate [21] $\frac{\partial r}{\partial t}$ can be written as follows:

$$
\begin{aligned}
J & =\frac{q_{c}}{(1+\theta)} \frac{\rho_{g}}{\rho_{l}} \sqrt{\frac{2 \sigma}{M_{m}^{3} \pi}} \exp \left(-\frac{4 \pi r_{c}^{3} \sigma}{3 K_{b} T_{g}}\right) \\
\frac{\partial r}{\partial t} & =\frac{p}{\rho_{l} h_{l g} \sqrt{2 \pi R T}} \frac{(\gamma+1)}{2 \gamma} C_{p}\left(T_{l}-T\right)
\end{aligned}
$$

In the above equations, $q_{c}$ is the condensation coefficient, $K_{b}$ is the Boltzmann constant, $M_{m}$ is single water molecule mass, $\sigma$ is the droplet surface tension, and $\theta$ is a nonisothermal correction factor that is given by the following relation:

$$
\theta=\frac{2(\gamma-1)}{(\gamma+1)}\left(\frac{h_{l g}}{R T}\right)\left(\frac{h_{l g}}{R T}-0.5\right)
$$

Here $h_{l g}$ is the latent heat of evaporation at pressure $p$ and $\gamma$ is the ratio of specific heat capacities.ps is the saturation pressure of water vapor at the local temperature $T$.

It is universally that for the high speed flow within the supersonic separator the turbulence intensity plays an important role in the flow pattern. Due to the concentrations of the liquid phase are dilute, the dispersed turbulence model is appropriate model for current calculation. The $k$ and $\varepsilon$ equations describing the Dispersed Turbulence Mode are as follows:

$$
\begin{array}{r}
\frac{\partial}{\partial t}\left((1-y) \rho_{g} k_{g}\right)+\nabla \cdot\left((1-y) \rho_{g} \vec{U}_{l} k_{g}\right)=(1-y) G_{k, \mathrm{~g}} \\
+\nabla\left((1-y) \frac{\mu_{t, \mathrm{~g}}}{\sigma_{k}} \nabla k_{g}\right) \\
-(1-y) \rho_{g} \varepsilon_{g}+(1-y) \rho_{g} \prod_{k_{g}}
\end{array}
$$

and

$$
\begin{array}{r}
\frac{\partial}{\partial t}\left((1-y) \rho_{g} \varepsilon_{g}\right)+\nabla \cdot\left((1-y) \rho_{g} \vec{U}_{l} \varepsilon_{g}\right)=(1-y) \rho_{g} \prod_{\varepsilon_{g}} \\
+\nabla\left((1-y) \frac{\mu_{t, g}}{\sigma_{k}} \nabla \varepsilon_{g}\right) \\
+(1-y) \frac{\varepsilon_{g}}{k_{g}}\left(C_{1 \varepsilon} G_{k, \mathrm{~g}}-C_{2 \varepsilon} \rho_{g} \varepsilon_{g}\right)
\end{array}
$$

Here $\prod_{k_{g}}$ and $\prod_{\varepsilon_{g}}$ epresent the influence of the dispersed liquid phases on the continuous gas phase, $\vec{U}$ is the gas phase-weighted velocity, and $G_{k, g}$ is the production of turbulent kinetic energy.

\section{Numerical Solver}

All calculations are run with help of the CFD code FLUENT 14.0. The gas and liquid phase conservation equations are discretized using finite-volume method. The non-equilibrium condensation model has been implemented into FLUENT Solver by UDF (user defined function). Droplet species equation solved by UDS (user defined Scalar) module include in the FLUENT Solver. Quick scheme is used to discretize convective terms, and coupled procedure is used to solve the momentum and energy equations simultaneously. First-order upwind schemes are set for density term. Second-order upwind schemes are selected for the necessary terms, such as turbulent kinetic energy, and turbulent dissipation rate.

\section{E. Calculation Domain and Boundary Conditions}

In order to give a successful demonstration of the flow field within the supersonic separator, the whole structure was chosen for the calculation domain, Table I listed the main geometrical data of the separator. Fig. 2 shows the three-dimensional calculation domain. The grid is intensified near the throat and swirling flow regions. The three-dimensional grids of the geometry were meshing with GAMBIT 2.4.6. Such meshing method is based on the check 
of grid independence. Preliminary test about grid independence of the computational domain showed that it is unnecessary to increase the number of the cells beyond the 1115982. To retain the calculation speed advantage coming with the use of a regular block-structure element, the multi-block technique was applied to make the concentration of grid density focused on the areas where significant phenomena were expected.

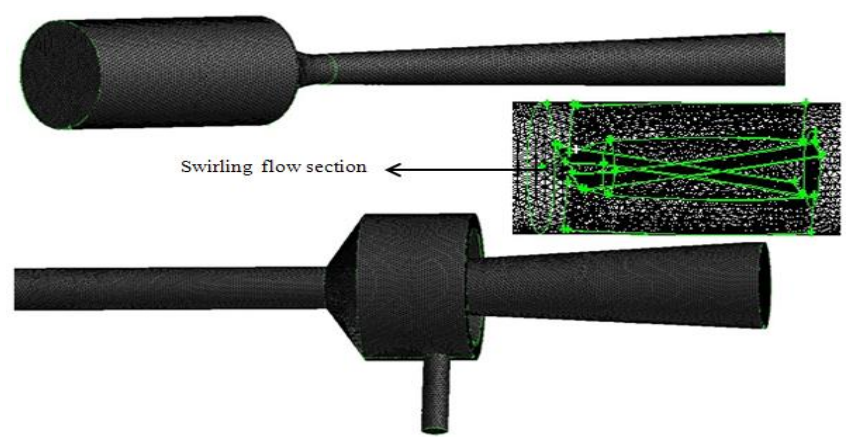

Fig. 2. Mesh partitioning strategy of the supersonic separator.

Boundary conditions in the supersonic separator are defined as follows:

1) At the separator entrance, compressed air with known pressure and total temperature are specified,
2) At the dry and wet outlet, pressure is assumed,

3) The wall of the supersonic separator is assumed adiabatic and no slip conditions are used for flow velocity components.

\section{F. Grid Independence Analysis \\ 1) Model verification}

Although the detailed numerical solution methods are introduced in the above paragraphs, it is necessary to validate whether these methods could be used to predict the complex swirling flow in a supersonic separator. However, as far as the present authors could know up to now, only a few sample results about the experimental test have been published in the open literature. So, in this paper, another supersonic separator that used in the previous experiment by Liu [9] was also simulated to verify the accuracy of the numerical model. For the verification case, the working fluid is mixture of air and water to keep the same material properties as the experiment conditions. Fig. 3 shows the comparison of the temperature distribution along the separator centerline between the simulations results and experiment. The comparison curve indicates that although some calculation data is slightly deviation compare to the experiment ones, there is still a great agreement between the two in the whole trend.

TABLE I: THE BASIC DIMENSIONS OF THE STRUCTURE

\begin{tabular}{ccccccccccc}
\hline \multicolumn{10}{c}{ TABLE I: THE BASIC DIMENSIONS OF THE STRUCTURE } \\
& $\begin{array}{c}d_{i} \\
(\mathrm{~mm})\end{array}$ & $\begin{array}{c}d_{t} \\
(\mathrm{~mm})\end{array}$ & $\begin{array}{c}d_{s} \\
(\mathrm{~mm})\end{array}$ & $\begin{array}{c}d_{h} \\
(\mathrm{~mm})\end{array}$ & $\begin{array}{c}L_{1} \\
(\mathrm{~mm})\end{array}$ & $\begin{array}{c}L_{2} \\
(\mathrm{~mm})\end{array}$ & $\begin{array}{c}L_{3} \\
(\mathrm{~mm})\end{array}$ & $\begin{array}{c}L_{4} \\
(\mathrm{~mm})\end{array}$ & $\begin{array}{c}L_{5} \\
(\mathrm{~mm})\end{array}$ & $\begin{array}{c}L_{6} \\
(\mathrm{~mm})\end{array}$ \\
\hline Computational model & 25 & 8 & 12 & 25 & 45 & 15 & 100 & 25 & 175 & 80 \\
\hline
\end{tabular}

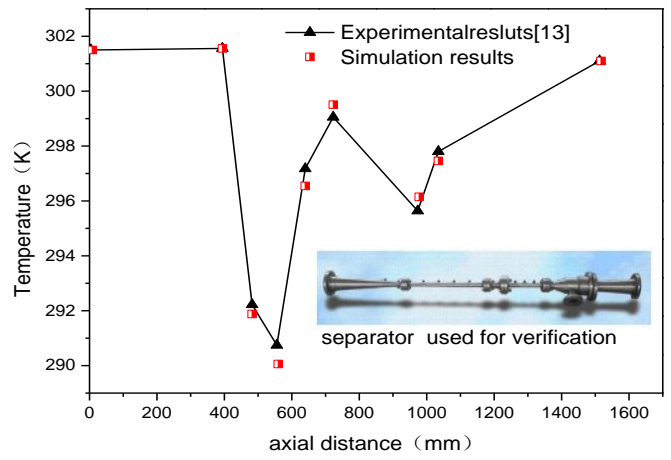

Fig. 3. Temperature comparison of the simulation results versus the experimental results.

\section{RESULTS AND DISCUSSIONS}

Bearing in mind that the working fluid is the mixture of the methane and water in the next section of this paper, the whole calculation structure has been given (Fig. 2). The calculation conditions are as follows: the inlet pressure is $0.45 \mathrm{MPa}$, the temperature is $300 \mathrm{~K}$, and the outlet static pressure is $0.25 \mathrm{MPa}$.

\section{A. Analysis of the Flow Field}

For the supersonic separator, the mainly driving force is pressure difference between the inlet and outlet (in this paper dry outlet and wet outlet keep the same pressure value). Fig. 4 shows the pressure contour on a cutting plane passing through the axial of the supersonic separator. As is apparent in Fig. 4 that along the whole supersonic separator at first the pressure decrease gradually when at the shock wave location the minimum pressure is only $0.2 \mathrm{MPa}$ then it jump abruptly to a bigger value, after the shock wave [22] location the pressure will not appear obviously change except the bifurcation point where the condensate droplets were removed.

In the supersonic separator, pressurized gas expands to low pressure will lead to low temperature and high speed. To complete the analysis of the mechanism of flow characteristic, Fig. 5-Fig. 7 depict the contour profile of velocity, temperature and Mach number on the plan passing through the axis, respectively. It is observed from Fig. 6 that the minimum temperature is $190 \mathrm{~K}$ appears in the divergent part of the Laval nozzle. Such a low temperature provides good condition for the water vapor condensation. Care must be taken with the formation of higher temperature at diffuser section that nearby the trailing section of the diffuser the temperature increase to $310 \mathrm{~K}$ which is higher than the inlet temperature. This is because the strong vortex effect generated by the centrifugal force will cause fiercely kinetic energy transfer [23].

It can be seen from the Fig. 5 and Fig. 7 that the maximum velocity will be $450 \mathrm{~m} / \mathrm{s}$ correspondingly the Mach number is 1.7. These conditions can maintain the gas flow in the supersonic state at the diverging part of the Laval nozzle and keep gas residence time in this section 
below two milliseconds to avoid extra hydrate inhibitors. Besides, at the trailing section of the swirling flow generator flow parameters include the pressure, temperature, velocity and Mach number will appear slightly obvious fluctuation.
In current operational condition, after the swirling flow generator temperature will increase while pressure, velocity and Mach number will decrease.

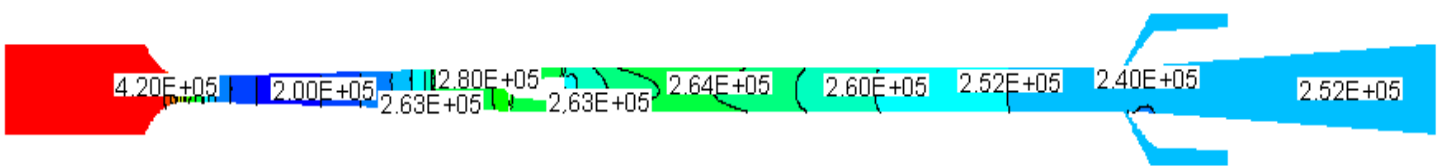

Fig. 4. Pressure distribution on the plane passing through the axis (Pa).

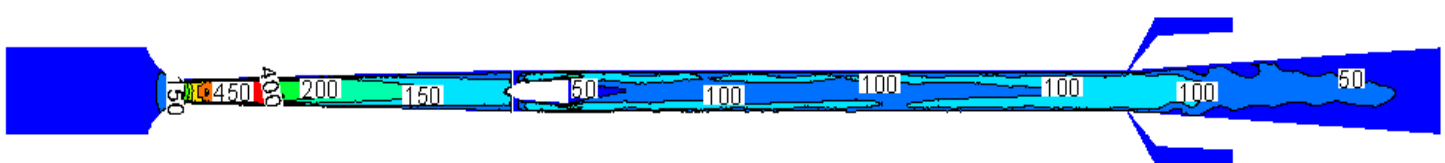

Fig. 5. Gas phase velocity distribution on the plane passing through the axis $(\mathrm{m} / \mathrm{s})$.

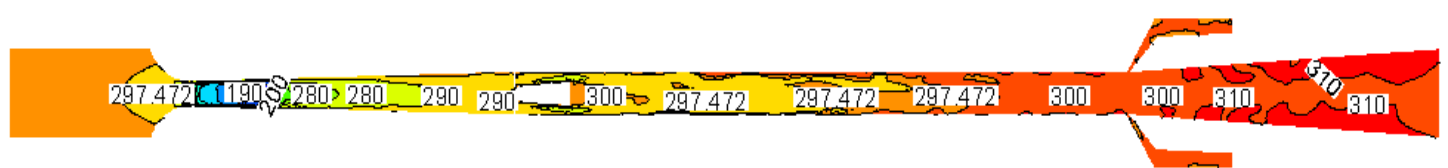

Fig. 6. Gas phase temperature distribution on the plane passing through the axis $(\mathrm{K})$.

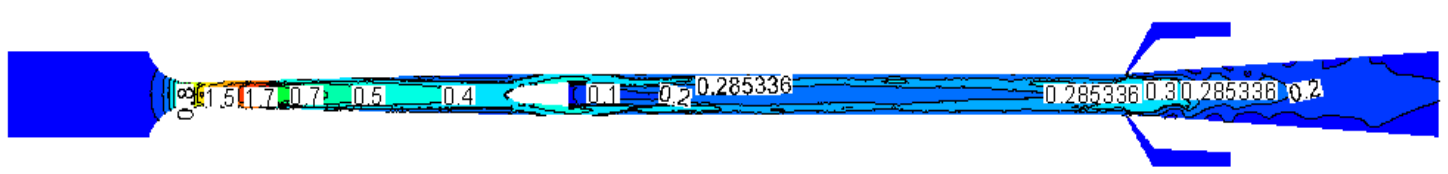

Fig. 7. Gas phase Mach number distribution on the plane passing through the axis.

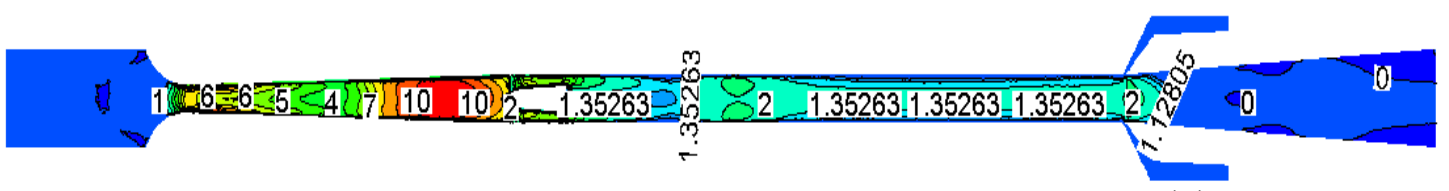

Fig. 8. Nucleation rate distribution on the plane passing through the axis $\left(\log J, J\left(\mathrm{~kg}^{-1} \mathrm{~s}^{-1}\right)\right)$.

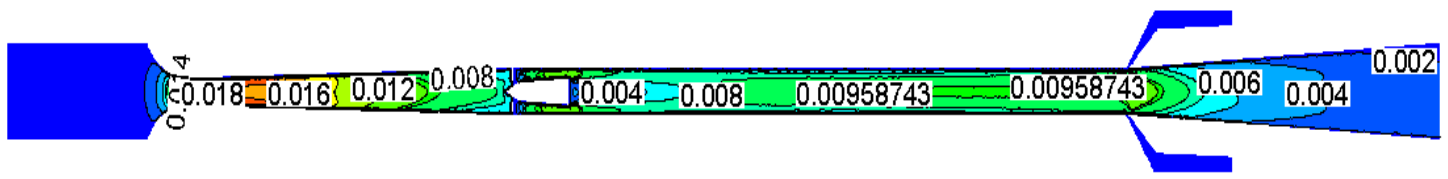

Fig. 9. Liquid fraction distribution on the plane passing through the axis.

Within the supersonic separator, the condensation phenomenon is another important process need to be demonstrated clearly. Thus, Fig. 8 presents the logarithmic nucleation rate contour under current operational condition, from this figure it can be known that nucleation mainly occurs at the diverging region of the Laval nozzle, especially at upstream of the swirling flow generator the nucleation rate reaches the maximum value $10 \times 10^{10} \mathrm{~kg}^{-1} \mathrm{~s}^{-1}$.

Through the above qualitative description of the flow field within the supersonic separator, it is reasonable to conclude that the thermodynamic phenomenon include pressure expansion, cryogenic process, supersonic region, shock wave location and spontaneous condensation have been reproduced. These further provide evidence that the numerical model established in this study is suitable for the full three-dimensional simulation on the flow characteristic of a supersonic separator.

According to the working principle of the supersonic separator one can conclude that swirling intensity in the straight section also play a significant role in satisfying its performance, so the swirling intensity can regard as one important factor used for evaluating centrifugal force which will cause the variation of the separation efficiency. Generally, higher swirling intensity will lead to more condensate separated to the walls form the main stream, and weaker swirling intensity will lower the separation efficiency. However, in current separator structure the swirling generator is located in the straight tube; the length of straight tube must have a great effect on the swirling intensity. When the length of straight tube is too long, strong swirling intensity will cause much more energy losses, too short length cannot separate the liquid thoroughly. Therefore, there may be an optimal value of swirl section for the best separation performance.

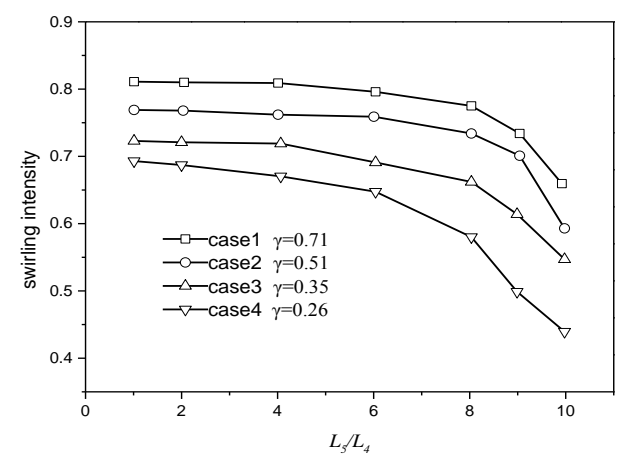

Fig. 10. Swirling intensity of the straight tube exit as a function of dimensionless parameter $L_{5} / L_{4}$ with different pressure loss ratio. 
The principle aim of the next section in this paper is investigate the relationship between the straight tube and swirling intensity. At first we defined two dimensionless parameters, swirling length ratio $L_{5} / L_{4}\left(L_{5}\right.$ is the length of straight tube, $L_{4}$ is the length of the swirling flow generator, in this paper is $25 \mathrm{~mm}$ ), swirling intensity $S$ which is the ratio of the tangential velocity to the axial velocity, respectively. The detailed form of the $S$ is defined as follows.

$$
S=\frac{u_{t}}{u_{w}}
$$

where $u_{t}$ is the area-weighted average tangential speed of the flow at the exit of the straight tube and $u_{w}$ denotes the area-weighted average axial speed at the exit of the straight tube. Besides, there need another factor to demonstrate the performance of supersonic separator, pressure loss ratio $\gamma$, which s defined as the ratio of the pressure loss $\Delta p$ of the supersonic separator to its inlet pressure $p_{\text {inlet }}$, that is,

$$
\gamma=\frac{\Delta p}{p_{\text {inlet }}}=\frac{p_{\text {inlet }}-p_{\text {outlet }}}{p_{\text {inlet }}}
$$

$P_{\text {outlet }}$ is pressure at gas outlet (both dry-outlet and wet-outlet are identical).

Drawing the important relationship between these dimensionless parameters help us to better understand the physics process of the problem within the supersonic separator. Fig. 10 shows the variation of swirling intensity obtained at the exit location of the straight tube versus the dimensionless parameter $L_{5} / L_{4}$ with different pressure loss ratio cases. It is observed that at the certain case 1 with the increase of the $L_{5} / L_{4}$ at the value ranges from 1 to 8 the swirling intensity decrease slightly, but when the $L_{5} / L_{4}$ exceed the value 8 the swirling intensity decrease fiercely. Under different pressure loss ratio cases the variation trend of these curves are slightly different. According to the different pressure loss ratio, by decreasing it, the critical value of $L_{5} / L_{4}$ will become smaller and the decrease trend of the swirling intensity is more obvious. These results leads to the conclusion that by increasing the $L_{5} / L_{4}$ in a certain range, the performance of the supersonic separator should be improved because of the increasing wall surface area for particles collection and extend of the resident time for gas/liquid separation. However, too high of the $L_{5} / L_{4}$ will cause more friction loss, impairing swirling separation effect. In this study, the optimal length of the straight tube should be 8 times the length of swirling flow generator at the pressure loss ratio case 1 and 6 times at the other pressure loss ratio cases.

\section{CONCLUSIONS}

A numerical analysis of supersonic separator has been established to analyze the flow pattern within the separator. The accuracy of the CFD simulations has been validated through comparison with experimental data obtained through literature. Based on the numerical model for the supersonic separator, the main conclusions are obtained and listed as follows:

1) The numerical method developed in this paper has a higher credibility for solving supersonic non-equilibrium condensation flow, and can predict the location of maximum droplet nucleation.

2) For the condensation flow within the supersonic separator, the obvious nucleation rate will occur at diverging section of the Laval nozzle, especially upstream the swirling flow generator, the maximum nucleation rate will $10 \times 10^{10} \mathrm{~kg}^{-1} \mathrm{~s}^{-1}$.

3) In the current study, the pressure loss ratio and the swirling length ratio $L_{5} / L_{4}$ have proved to play a great role in improving the swirling separation effect. It was obtained that when the pressure loss ratio is 071 the optimal $L_{5} / L_{4}$ value should be 8 , with the decrease of the pressure loss ratio the optimal $L_{5} / L_{4}$ should be decreased correspondingly.

\section{ACKNOWLEDGEMENTS}

This work was supported by the National Natural Science Foundation of China (Grant No. 50676002) and the Research Fund for the Doctoral Program of Higher Education (Grant No. 20040005008).

\section{REFERENCES}

[1] Y. Song et al., "The status of natural gas hydrate research in China: A review," Renewable and Sustainable Energy Reviews, vol. 31, pp. 778-91, 2014.

[2] M. Betting et al., "Supersonic separator apparatus and method," US Patent 6524368b2, 2003.

[3] J. M. Brouwer, G. Bakker, H-J. Verschoof, and D. H. Epsom, "Twister supersonic gas conditioning-first commercial offshore experience," GPA paper, 2004

[4] P. B. Machado, J. G. M. Monteiro, J. L. Medeiros, H. D. Epsom, O. Q. F. Araujo, "Supersonic separation in onshore natural gas dew point plant," Journal of Natural Gas Science and Engineering, vol. 6, pp. 43-49, 2012.

[5] P. Schinkelshoek and H. Epsom, "Supersonic gas conditioning-low pressure drop twister for NGL recovery," presented at the Offshore Technology Conference.

[6] K. Y. Gu, Z. L. Liu, H. W. Liu, H. D. Liu, Y. M. Gu, and C. Wang, "Vortex gas purification and separation device," China Patent 1602992, 2005.

[7] P. Schinkelshoek and H. D. Epsom, "Supersonic gas conditioning -introduction of the low pressure drop twister," GPA Europe 2005.

[8] Y. Alf et al., "Supersonic nozzle efficiently separates natural gas components," Oil \& Gas Journal, vol. 5, pp. 53-58, 2005.

[9] H. W. Liu, Z. L. Liu, Y. X. Feng, K. Y. Gu, and T. M. Yan, "Characteristics of a supersonic swirling dehydration system of natural gas," Chinese Journal of Chemical Engineering, vol. 13, pp. 9-12, 2005.

[10] J. W. Ming, L. Z. Liang, L. H. Wei, Z. X. Jun, and Z. Jian, "Field test study of new type supersonic dehydration facilities in natural gas purification plant," Natural Gas Industry, vol. 28, pp. 136-138, 2008.

[11] M. M. Malyshkina, "The structure of gasdynamic flow in a supersonic separator of natural gas," High Temperature, vol. 46, pp. 69-76, 2008.

[12] M. M. Malyshkina, "The procedure for investigation of the efficiency of purification of natural gases in a supersonic separator," High Temperature, vol. 48, pp. 244-250, 2010.

[13] W. M. Jiang, Z. L. Liu, H. W. Liu, H. Z. Pang, and L. L. Bao, "Influences of friction drag on spontaneous condensation in water vapor supersonic flows," Science in China Series E: Technological Sciences, vol. 52, pp. 2653-2659, 2009.

[14] L. L. Bao, Z. L. Liu, H. W. Liu, W. M. Jiang, M. Zhang, and J. Zhang, "Phase equilibrium calculation of multi-component gas separation of supersonic separator," Science China Technological Sciences, vol. 53, pp. 435-443, 2010.

[15] E. Jassim, M. A. Abdi, and Y. Muzychka, "Computational fluid dynamics study for flow of natural gas through high-pressure supersonic nozzles: part 2, nozzle geometry and vorticity," Petroleum Science and Technology, vol. 26, pp. 1773-1785, 2008. 
[16] E. Jassim, M. A. Abdi, and Y. Muzychka, "Computational fluid dynamics study for flow of natural gas through high-pressure supersonic nozzles: part 1, real gas effects and shockwave," Petroleum Science and Technology, vol. 26, pp. 1757-1772, 2008.

[17] S. H. R. Shooshtari and A. Shahsavand, "Reliable prediction of condensation rates for purification of natural gas via supersonic separators," Separation and Purification Technology, vol. 116, pp. 458-470, 2013.

[18] C. Wen, X. W. Cao, Y. Yang, and J. Zhang, "Evaluation of natural gas dehydration in supersonic swirling separators applying the discrete particle method," Advanced Powder Technology, vol. 23, pp. 228-233, 2012.

[19] Q. Ma et al., "Performance of inner-core supersonic gas separation device with droplet enlargement method," Chinese Journal of Chemical Engineering, vol. 17, pp. 925-933, 2009.

[20] Y. Yang and S. Shen, "Numerical simulation on non-equilibrium spontaneous condensation in supersonic steam flow," International Communications in Heat and Mass Transfer, vol. 36, pp. 902-907, 2009.

[21] Y. Yang, S. Shen, T. Kong, and K. Zhang, "Numerical investigation of homogeneous nucleation and shock effect in high-speed transonic steam flow," Heat Transfer Engineering, vol. 31, pp. 1007-1014, 2010.

[22] C. Wen, X. W. Cao, Y. Yang, and W. L. Li, "Numerical simulation of natural gas flows in diffusers for supersonic separators," Energy, vol. 37, pp. 195-200, 2012.

[23] M. Farzaneh-Gord and M. Kargaran, "Recovering energy at entry of natural gas into customer premises by employing a counter-flow vortex tube," Oil Gas Sci. Technol.-Rev IFP Energies Nouvelles, vol. 65, pp. 903-912, 2010.

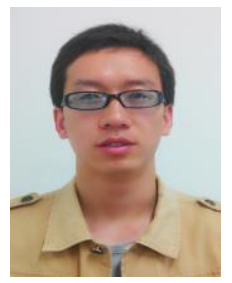

Liu Xingwei was born in Hebei Province in 1985 Presently he is study in Beijing University of Technology, he is a candidate for doctor degree of thermal engineering; he received bachelor degree of building environment and equipment engineering in Tangshan college. Currently, he is specialized in numerical simulation technology and optimization of the flow and heat transfer and supersonic separation technology of natural gas.

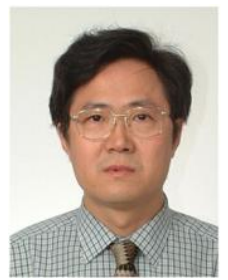

Liu Zhongliang was born in Hebei Province in 1958. He is a professor in thermal fluids \& energy engineering, at College of Environmental \& Energy Engineering, Principal of Beijing-Dublin International College at BJUT, Beijing University of Technology, Pingleyuan 100, Chaoyang District, Beijing 100124, China. His research direction include strengthen the heat transfer and its application in high and new technology, environmental energy technology research and development, numerical simulation technology and optimization of the flow and heat transfer et al.

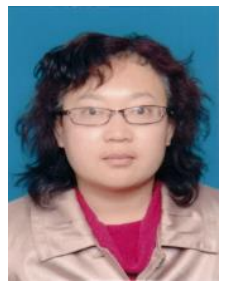

Li Yanxia was born in Shandong Province. She received master degree in Beijing University of Technology in July 2003. Then she received doctor degree in Beijing University of Technology in July 2012. Since June 2003, she has been a lecturer and teaching assistance at Beijing University of Technology, College of Environmental and Energy Engineering. Her main research direction is environmental energy research and development of high and new technology. 



\section{Renewable Energy Utilizations}


